\title{
REGULATING NITROGEN POLLUTION WITH RISK-AVERSE FARMERS UNDER HIDDEN INFORMATION AND MORAL HAZARD by
}

\author{
Philippe Bontems and Alban Thomas
}

\author{
Corresponding author: Philippe Bontems \\ INRA \\ Université des Sciences Sociales de Toulouse \\ 21 Allée de Brienne \\ 31000 Toulouse, France \\ Email: bontems@toulouse.inra.fr
}

September 27, 2004 


\section{REGULATING NITROGEN POLLUTION WITH RISK-AVERSE FARMERS UNDER HIDDEN INFORMATION AND MORAL HAZARD}

\section{PHILIPPE BONTEMS AND ALBAN THOMAS}

We consider a model of pollution regulation for a risk-averse farmer involving hidden information, moral hazard and risk sharing. The representative farmer faces a production risk originating from nitrogen leaching, and privately observes the soil capacity in retaining nitrogen only after the regulation contract is signed. The latter specifies a transfer and a nitrogen quota, whose decomposition by the farmer among different production stages is unknown to the regulator. We first characterize the optimal solution to the regulator's problem. The sequential decision model is estimated on French crop production data and the results are used to calibrate and simulate the optimal contract.

Keywords: asymmetric information, mechanism design, moral hazard, non point source pollution, risk aversion, split nitrogen application

Philippe Bontems and Alban Thomas are both affiliated with the Institut National de la Recherche Agronomique (INRA), Toulouse, France.

An earlier version of this article has been circulated under the title "Optimal Regulation under Asymmetric Information and Risk Aversion, with an Application to Pollution Control". We thank Thierry Magnac and Catarina Roseta-Palma for their comments on a previous version. We also thank participants and discussants at the following conferences and seminars: EAERE 2001 (Southampton), AAEA 2001 (Chicago), CREE 2001 (Brock U.), CSERGE (London), AIELF 2001 (Montréal), Conference on Risk and Uncertainty in Environmental and Resource Economics 2002 (Wageningen), the $3^{\text {rd }}$ Annual Workshop on the Economics of Contracts in Agriculture 2003 (Copenhague). Financial support from INRA AIP "Théorie des Contrats" is gratefully acknowledged. Any remaining errors are the authors'. 


\section{REGULATING NITROGEN POLLUTION WITH RISK-AVERSE FARMERS UNDER HIDDEN INFORMATION AND MORAL HAZARD}

The increased concern for nitrate pollution in water from agriculture has led the way to a number of theoretical propositions for environmental protection. Two approaches are basically encountered in the literature, the first dealing with taxation or quota on nitrogen (see among others $\mathrm{Wu}$ and Babcock), and the second addressing the issue of nonpoint source pollution from a team-regulation perspective (see e.g. Segerson). Two aspects of agricultural activity are important to account for when designing optimal contracts for regulating nitrate pollution from agriculture. First, farmer risk aversion is essential in assessing correctly the choice of inputs and cropping practices; omitting attitude towards risk may result in incorrect interpretation (see e.g. Babcock and Hennessy, Babcock, Lambert and Leathers and Quiggin). Second, nitrogen application is a complex process for which farmers have a large degree of flexibility; in particular, split application depending on weather conditions may be as important as the total nitrogen supplied for crop yield (Feinerman, Choi and Johnson, Huang, Uri and Hansen). Moreover, split nitrogen application has a significant impact on groundwater pollution.

Under risk aversion, split nitrogen application comes as a practical means of production risk hedging (see Bontems and Thomas). Since actual split nitrogen applications are unobservable to the environmental regulator, farmers have to be regulated through a contract scheme based on total nitrogen use. A major difficulty in our framework originates from the fact that the regulator does not have accurate information on farmer production characteristics. In particular, the soil nitrogen run-off potential is private information to the farmer, but this parameter is only observed by him after the contract is signed. This is because significant nitrogen leaching occurs between the first nitrogen application (end of winter) and the subsequent applications in late spring (Feinerman, Choi and Johnson).

Optimal contract schemes when the relationship between a regulator and an agent is 
subject to asymmetric information (in the form of a private-information parameter to the agent) are now widely documented in the literature (see Laffont and Tirole for a synthesis). In these models, private information to the agent may be used for strategic purposes in the relationship with the principal, but it is most of the time assumed that this parameter is observed to the agent before the contract is signed. Consequently, the regulator has to design a contract mechanism consistent with incentive-compatibility and individual-rationality constraints (the contract must produce truthful revelation of the agent's type, and the agent must find it profitable to accept the contract).

However, in a number of situations in agriculture, it may be the case that the farmer does not observe her private-information parameter before engaging in a contract-based relationship with the environmental regulator. Consequently, when signing a contract, the agent faces uncertainty which is not resolved yet. Informational asymmetries of this type would be of no consequence for the principal if the agent were risk neutral or if there were no limited liability (or ex-post individual rationality) constraints. ${ }^{1}$ However, when the agent is riskaverse, the task of the regulator is more difficult, as inducing the appropriate risk-sharing is required in addition to the informational constraints mentioned above. The situation is even more complicated when some decisions taken by the agent during the regulation period are non contractible and yield to moral hazard problems. The purpose of this article is to take a look at such situations by considering a principal - risk-averse agent model, and to illustrate the complex trade-off between the distortions due to hidden information, moral hazard and risk sharing. More precisely, the task of the principal here is to find an incentive scheme designed for a risk-averse agent whose non contractible actions are taken after the date of the contract, some of those before the private-information parameter is observed by the agent, and some after.

Our framework has similarity with existing models of regulation under hidden information and with risk-averse agents. More precisely, Salanié and Laffont-Rochet provide a formal analysis of similar cases, in producer-retailer contract for the first paper and in the context of 
public regulation for the second one. There, it is shown that a finite risk aversion implies that optimal mechanisms are less efficient. In this case, distortions of agent actions are greater and agent information rents are socially more costly and thus diminished by the principal. Our analysis differs from these two papers in that the regulated agent takes here an action (in the first stage of the production process) when the uncertainty is still unresolved. As this decision is not observable and thus non contractible by the regulator, the optimal contract has to induce the agent to take the optimal decision on the first action (moral hazard) as well as inducing truthful revelation of type. It also has to achieve an optimal risk sharing between the principal and the agent: the regulator being risk neutral, he has to absorb as much risk as possible through the contract design.

In this article, we first characterize the optimal regulatory scheme in the context of pollution control by an environmental agency, where the agent is a risk-averse farmer facing production risk because of nitrogen leaching, and the private-information parameter measures the soil capacity in retaining nitrogen. Then, in order to simulate the optimal contract, the farmer's sequential decision model is estimated for a sample of French corn producers. This enables us to estimate the substitutability pattern between nitrogen at different stages. Estimation results are then used to calibrate and simulate the optimal contract. This is important for assessing the sensitivity of the optimal regulatory scheme to farmer risk preferences, the social damage of pollution or the shadow cost of public funds.

Although the contract-based regulation scheme is purely virtual, simulation experiments are nevertheless useful to better understand the role of technological constraints in designing more sophisticated environmental policies aimed at farmers. Furthermore, it is interesting to compare our regulatory design with a selection of already-existing policies to control for nonpoint source pollution. In the Netherlands, a tax system on nitrogen carryover has been imposed on cattle farmers since 1998. Farmers report their nitrogen expenditures to the central environmental authority which then computes index of nitrogen carryover per hectare. Based on the latter, the regulatory agency allows for a given nitrogen surplus, above which 
farmers are taxed (Polman and Thijssen). In France, a similar system was proposed in 2001, but the government decided to postpone its implementation. At the European level, the 1991 Directive on Nitrate requires state members to design a chart of best agricultural practices, which is mandatory for Environmental Sensitive Areas (ESA), and voluntary for others. In sensitive areas, public authorities can impose restrictions on nitrogen use, which then become part of a command and control-type policy. Nevertheless, most state members have experienced difficulties in abiding by the European agenda (France has been sued by the European Court of Justice in March 2001 because of its delay in implementing the Nitrate Directive).

We first present the model of production under risk conditions and describe the agency problem, and we characterize the optimal solution to the agency problem under asymmetric information. We then present the data used in the application and the econometric estimation used to calibrate the sequential production process. Finally, we conduct a simulation experiment to compute optimal nitrogen paths for the no-regulation, perfect information, risk neutrality and risk aversion cases.

\section{The model}

Consider a representative farmer engaged in crop production which requires the use of a potentially-polluting input (nitrogen fertilizer). As it is well known in empirical agricultural production analysis, farmers often hedge against risk due to nitrogen leaching by the use of a split nitrogen application strategy (see e.g. Huang, Uri and Hansen, Feinerman, Choi and Johnson). This strategy tries to compensate for the nitrogen loss that may occur during the plant growth period, because of excess rainfall. We first present the agricultural production model, by characterizing the actions taken during the successive stages in the production process (see Bontems and Thomas for a similar dynamic framework).

Agricultural production and pollution 
The basic economic model of nitrogen application under risk aversion consists in sequential decisions for the farmer. The source of uncertainty is the extent of nitrogen leaching during the growing season. At date 1 (late winter or early spring), the producer has to make a fertilizer primary application (denoted by $X$ ), knowing the initial stock $A$ of nitrogen in soil. At date 2, the farmer learns the intensity of nitrogen runoff, which applies to the total nitrogen stock composed of $A$ and $X$. Finally, at date 3 (growing season), the producer makes a complementary fertilizer application (side-dressing), denoted $Y$ (see Boswell, Meisinger and Case, Dilz, Meisinger and Randall, and Pierce, Shaffer and Halvorson for agronomical considerations). The total level of input consists of the sum of the two applications and is denoted $Z=X+Y$.

Viewing the problem in terms of sequential decisions under risk conditions allows us to model nitrogen availability to the plant as follows. Let $N_{t}$ denote total nitrogen available at time $t, A=N_{0}$ initial nitrogen stock before planting, and $R$ crop yield. The nitrogen uptake by the plant is assumed to be a fraction $\gamma$ of nitrogen available. The nitrogen run-off parameter is denoted $\alpha$, i.e. $\alpha$ is the fraction of nitrogen not subject to run-off and available to the crop. The parameter $\alpha$ belongs to the set $[\underline{\alpha}, \bar{\alpha}]$ which is included in the interval $[0,1]$.

Let $\tilde{X}$ and $\tilde{Y}$ represent nitrogen up-take by the plant, i.e. the useful input level to grow crop. With parameters introduced above, we have:

$$
\tilde{X}=\gamma \alpha(A+X) \quad \text { and } \quad \tilde{Y}=\gamma\left(N_{1}+Y\right),
$$

where $N_{1}=(1-\gamma) \alpha(A+X)$.

The successive nitrogen levels in soil are $N_{0}=A, N_{1}=(1-\gamma) \alpha(A+X)$ and $N_{2}=$ $(1-\gamma)\left(N_{1}+Y\right)$. The yield $R$ is a function of past nitrogen stocks and successive nitrogen applications:

$$
R=F(\tilde{X}, \tilde{Y})=F\left(\gamma \alpha(A+X), \gamma\left(N_{1}+Y\right)\right)
$$

We assume that $F$ is increasing and concave in $(\tilde{X}, \tilde{Y})$ together with $\frac{\partial^{2} F}{\partial \tilde{X} \partial \tilde{Y}}<0$, which means that first and second nitrogen up-take are substitutes in the production function. 
Given a price system $(p, w)$ for respectively output and nitrogen input, the ex-post profit $\Pi$ without any regulation may be written as follows ${ }^{2}$ :

$$
\Pi=p F(\tilde{X}, \tilde{Y})-w(X+Y)
$$

It is convenient for the following analysis to rewrite yield $R$ as a function of actual primary application $X$, total application $Z$ and parameter $\alpha$ :

$$
R=F(\tilde{X}, \tilde{Y}) \equiv f(\alpha, X, Z)
$$

Note that it is equivalent for the analysis to consider the pair $(X, Y)$ or the pair $(X, Z)$. Given our assumptions on $F($.$) , the function f$ is such that $f_{\alpha}>0, f_{\alpha Z}<0$ which means that production strictly increases in the fraction $\alpha$ of nitrogen not subject to run-off and that the marginal productivity of total nitrogen $Z$ strictly decreases in $\alpha .^{3}$ Also, the function $f$ is concave in $Z$ and $X$ with $f_{Z Z}<0, f_{X X}<0$. Note that while concavity of $F$ implies the former we assume the latter. Furthermore, we assume that $X$ and $Z$ are complements in the production function $\left(f_{X Z}>0\right)$, which is actually satisfied in our empirical application. This implies that a reduction in total nitrogen applied would also induce a reduction in the first application $X$.

The ex-post profit is now

$$
\Pi(\alpha, X, Z)=p f(\alpha, X, Z)-w Z
$$

The farmer's problem is to maximize expected profit if she is risk-neutral, or to maximize the expected utility of profit if she is risk-averse. In the latter case, the farmer's problem would be

$$
\max _{X} \int \max _{Z}[U(p f(\alpha, X, Z)-w Z)] d G(\alpha) \quad \text { s.t. } \quad 0 \leq X \leq Z
$$

where $U($.$) (with U^{\prime}()>0,. U^{\prime \prime}()<$.0 ) is the Von Neuman-Morgenstern utility function and $G($.$) is the probability distribution function for private information parameter \alpha$. Note that by assumption $\alpha$ is the only random component in the profit function, its randomness being 
understood as associated with the fact that decision on $X$ is taken before the true value of the private information parameter $\alpha$ is observed. ${ }^{4,5}$

There is an on-going debate in the literature concerning the role of risk aversion on fertilizer input use. Babcock recognizes the paradox that farmers seem to over-apply nitrogen fertilizer although they perceive it as a risk-increasing input. Ramaswami presents a weak condition on technology for signing marginal risk premium associated with inputs, and associate the risk-increasing property of an input with the marginal variance with respect to this input, in the Just and Pope case. Finally, Hennessy and Roosen test for the role of risk aversion on fertilizer use by U.S. corn producers. Their results suggest that risk-averse farmers apply less nitrogen levels than risk-neutral ones, and that an increase in risk aversion may reduce input use (although this last result is less robust). In our framework, let us denote $X^{\circ}$ and $Z^{\circ}\left(\alpha, X^{\circ}\right)$ the optimal solutions of program (1) and denote $\Pi^{\circ}=\Pi\left(\alpha, X^{\circ}, Z^{\circ}\left(\alpha, X^{\circ}\right)\right)$. If further, an increase in the fraction of nitrogen not subject to run-off raises the marginal productivity of $X$, i.e. $\partial^{2} \Pi^{\circ} / \partial \alpha \partial X=p f_{\alpha X}\left(\alpha, X^{\circ}, Z^{\circ}\left(\alpha, X^{\circ}\right)\right)>0$, then an increase in $X$ also renders the profit function more sensitive to the source of risk. Consequently, the risk averse farmer might reduce the first application level $X$ compared to the risk neutral one (see Appendix 2 for a necessary and sufficient condition). Moreover, as $X$ and $Z$ are complements $\left(f_{X Z}>0\right)$, if the risk averse farmer reduces $X$, he also diminishes the total level of nitrogen applied $Z$ whatever the realization of the risk. This plausible scenario is actually met in our empirical application presented below.

In any case, whatever the farmer preferences and the source of uncertainty, regulation is needed on the grounds that producers do not account for negative external effects of their nitrogen applications on the environment. This is of course particularly relevant when the nitrogen application rate is so high as to contaminate groundwater sources. We assume here that environmental damage only originates from excessive levels of $X$. Let $D($.$) denote the$ damage function, which is increasing and strictly convex in the quantity of nitrogen subject to leaching, $(1-\alpha)(A+X)$. 


\section{The regulator problem}

Consider now the problem of a risk-neutral regulatory agency engaged in a principal-agent relationship with the farmer. The goal of the principal is to maximize expected net social surplus associated with production, where the main policy instrument is a contract to be signed between the two parties. This contract specifies a level of input $Z$ and a transfer $T$ from the agency to the farmer. A crucial assumption in this case is that the contract is signed before any action the farmer might take, and importantly, before parameter $\alpha$ is observed to the producer. The transfer is given to the farmer at the end of the game. The nature of the contract is motivated by the fact that the agency may not be able to discriminate $X$ from the quota $Z$, but the latter is nevertheless verifiable.

At the time of contracting, both the agency and the farmer have a common prior on parameter $\alpha$, which is represented by the distribution function $G($.$) and g($.$) a density on$ the interval $[\underline{\alpha}, \bar{\alpha}]$. We assume that the agency can only get information on $\alpha$ through communication with the farmer and observes only total level $Z$. At the beginning of the regulatory game, the agency offers a menu of contracts $(T(\hat{\alpha}), Z(\hat{\alpha}))$ contingent on a message $\hat{\alpha} \in[\underline{\alpha}, \bar{\alpha}]$ sent later by the farmer when the uncertainty upon $\alpha$ is resolved. If the farmer refuses to contract, the game ends and the farmer is not allowed to produce. If the farmer accepts the menu of contracts, then he privately learns the true value of $\alpha$ before action $Y$ is taken but after the decision on $X$. He is then required to choose a particular contract in the menu by sending his message $\hat{\alpha}$ and hence he commits to a total input level $Z(\hat{\alpha})$ in exchange of the transfer $T(\hat{\alpha})$. Consequently, the type- $\alpha$ farmer who announces to be of type $\hat{\alpha}$ get the following (ex post) profit:

$$
\Pi(\alpha, \widehat{\alpha})=p f(\alpha, X, Z(\widehat{\alpha}))-w Z(\widehat{\alpha})+T(\widehat{\alpha}) .
$$

The timing of the game is depicted in figure 1.

From the revelation principle, there is no loss of generality in only paying attention 
to direct revelation mechanisms, i.e. mechanisms that induce the farmer to announce his true type for any type. As the communication between the agency and the producer must take place after the uncertainty upon $\alpha$ is resolved for the farmer, incentive compatibility constraints write: ${ }^{6}$

$$
\forall \alpha, \forall \widehat{\alpha}, \quad \Pi(\alpha) \equiv \Pi(\alpha, \alpha) \geq \Pi(\alpha, \widehat{\alpha})
$$

Because the level of $X$ is unobservable by the agency, the latter has to take into account a moral hazard constraint which states that whatever the contract is, $X$ should be optimal for the farmer:

$$
X \in \arg \max _{X \leq Z(\alpha)} \int_{\underline{\alpha}}^{\bar{\alpha}} U(\Pi(\alpha)) d G(\alpha) .
$$

We also assume that the regulator is exogenously constrained to offer a minimum profit whatever the realized value of $\alpha$, because of infinite risk aversion below zero (or limited liability constraints). We normalize the reservation utility of the farmer to zero and ex-post participation constraints thus read: ${ }^{7}$

$$
\Pi(\alpha) \geq 0, \forall \alpha \text {. }
$$

We now have to consider the objective function of the agency. It seeks to maximize the expected consumer surplus net of the social cost of the transfer $T$ paid to the farmer, plus the certainty equivalent of farmer's profit. Let $S($.$) denote the consumer surplus function which$ is concave. It also incorporates the damage function associated with nitrogen leaching, $D($.$) .$ The function $S($.$) is thus written as:$

$$
S(\alpha, X, Z(\alpha))=V[f(\alpha, X, Z(\alpha))]-p f(\alpha, X, Z(\alpha))-D((1-\alpha)(A+X)),
$$

where $V($.$) represents the gross consumer surplus for the agricultural good.$

The social cost of public transfers between the regulator and the farmer is assumed strictly positive, with an opportunity cost of public funds equal to $\lambda$. Finally, let $C E(\Pi)=$ 
$U^{-1}\left[E_{\alpha} U(\Pi(\alpha))\right]$ denote the certainty equivalent of $\Pi$, where $E_{\alpha}$ is the expectation operator with respect to the distribution of $\alpha$.

The agency's problem can now be written as

$$
\max _{X, Z(.), T(.)} \int_{\underline{\alpha}}^{\bar{\alpha}}[S(\alpha, X, Z(\alpha))-(1+\lambda) T(\alpha)] d G(\alpha)+C E(\Pi),
$$

subject to (IC), (MH), (IR), $0 \leq X \leq Z(\alpha), \Pi(\alpha)=p f(\alpha, X, Z(\alpha))-w Z(\alpha)+T(\alpha)$.

\section{Characterization of the optimal contract under perfect information}

At this point of the discussion, it is worth investigating the optimal solution under perfect information, that is when the agency is able to observe both $X$ and parameter $\alpha$. Ignoring both (IC) and (MH) in the program above, we eliminate the transfer $T$ and we rewrite the problem as:

$$
\begin{array}{r}
\max _{X, Z(.), \Pi(.)} \int_{\underline{\alpha}}^{\bar{\alpha}}[S(\alpha, X, Z(\alpha))-(1+\lambda)(\Pi(\alpha)-p f(\alpha, X, Z(\alpha))+w Z(\alpha))] d G(\alpha) \\
+C E(\Pi),
\end{array}
$$

s.t. (IR), $0 \leq X \leq Z(\alpha)$.

Maximising expected welfare over $X$ and $Z($.$) gives the following first order conditions$ (for interior solutions denoted $X^{P I}$ and $\left.Z^{P I}\right){ }^{8}$

$$
\begin{gathered}
(1+\lambda) \int_{\underline{\alpha}}^{\bar{\alpha}} p f_{X}\left(\alpha, X^{P I}, Z^{P I}(\alpha)\right) d G(\alpha)=\int_{\underline{\alpha}}^{\bar{\alpha}} D_{X}\left((1-\alpha)\left(A+X^{P I}\right)\right) d G(\alpha), \\
p f_{Z}\left(\alpha, X^{P I}, Z^{P I}(\alpha)\right)=w,
\end{gathered}
$$

indicating that under perfect information the optimal $X$ should equalize the marginal private benefit weighted by the social cost of public funds $(1+\lambda)$ with the expected marginal damage. Moreover, the marginal private benefit of total nitrogen use $Z$ is equal to its private cost $w$. Because $f_{\alpha Z}<0$ and $f_{Z Z} \leq 0$, it is easy to check that total nitrogen use $Z^{P I}$ decreases in $\alpha$.

Note that, conditional on $X$, the private optimum and the social optimum under perfect information concerning $Z$ coincide. Hence, given that $X$ is observable, regulating $Z$ is not 
actually needed. However, as the farmer does not take into account the social damage $D$ in the absence of regulation, he does not apply in general the socially optimal amount of $X$, and consequently the private choice of $Z$ described by (1) does not coincide with the social optimum given by (3). As the sign of $f_{X Z}$ is constant and positive in our empirical application, $X$ and $Z$ are complements. Consequently, if the farmer applies too much of $X$ absent any regulation, he also applies too much of total nitrogen $Z$.

Finally, any type of farmer earns no rent at the optimum, that is, $\Pi^{P I}(\alpha)=0, \forall \alpha$. To see that (IR) is binding at the optimum, assume that it is not. Then, the derivation of the principal's objective with regards to $\Pi(\alpha)$ yields to the following first order condition:

$$
\left[\frac{U^{\prime}\left(\Pi^{P I}(\alpha)\right)}{U^{\prime}\left(C E\left(\Pi^{P I}\right)\right)}-(1+\lambda)\right] g(\alpha)=0 .
$$

This means that the marginal utility of profit is constant for every $\alpha$. As the principal is risk neutral he should absorb all the risk. However, this also means that $\Pi^{P I}(\alpha)$ is equal to some constant $\pi$, and as $C E\left(\Pi^{P I}\right)=\Pi^{P I}$, this contradicts (4) as long as $\lambda>0$. This also contradicts the fact that (IR) is not binding. To conclude, in any case, the regulator places every farmer exactly at his reservation utility level.

An important feature of the optimal regulatory scheme under perfect information is that it does not depend on the risk preferences of the farmer. Moreover, it is obvious that, as long as $X$ is observable, there is no need for the regulator to control $Z$ which is chosen optimally by the agent. As will be clear below, these features of the optimal policy are not robust to the introduction of imperfect information.

\section{Characterization of the optimal contract under imperfect information}

Returning to the situation of imperfect information, it is clear that the complexity of incentives constraints does not allow us to solve program (P1) directly. However, we indicate in Appendix 4 how to transform it into an optimal control problem on which we apply usual optimal control techniques. We gather in the following proposition some properties of the 
optimal separating contract.

Proposition 1 Assuming an interior solution for $X$ and $Z$, an optimal separating contract is such that:

(i) $Z$ is given by the equality between the private marginal benefit and the virtual marginal cost,

$p f_{Z}(\alpha, X, Z(\alpha))=w-\frac{\kappa}{(1+\lambda)} U^{\prime}(\Pi(\alpha)) p f_{X Z}(\alpha, X, Z(\alpha))-\frac{\nu(\alpha)}{(1+\lambda) g(\alpha)} p f_{\alpha Z}(\alpha, X, Z(\alpha))$,

where $\nu(\alpha)$ is the shadow price of incentive compatibility constraints and $\kappa$ is the shadow price of the moral hazard constraint, with

$\left\{\begin{array}{l}\nu(\alpha)=-(1+\lambda)(1-G(\alpha))+\int_{\alpha}^{\bar{\alpha}} \frac{U^{\prime}(\Pi(u))}{U^{\prime}(C E(\Pi))} g(u) d u+\kappa \int_{\alpha}^{\bar{\alpha}}\left\{p f_{X}(u, X, Z(u)) U^{\prime \prime}(\Pi(u))\right\} g(u) d u, \\ \kappa=-\frac{\int_{\alpha}^{\bar{\alpha}}\left\{\left(S_{X}+(1+\lambda) p f_{X}\right) g+\left(\int_{\alpha}^{\bar{\alpha}}\left\{\frac{U^{\prime}(\Pi(u))}{U^{\prime}(C E(\Pi))}-(1+\lambda)\right\} g(u) d u\right) p f_{\alpha X}\right\} d \alpha}{\int_{\underline{\alpha}}^{\bar{\alpha}}\left\{\left(\int_{\alpha}^{\bar{\alpha}}\left\{p f_{X}(u, X, Z(u)) U^{\prime \prime}(\Pi(u))\right\} g d u\right) p f_{\alpha X}+U^{\prime}(\Pi) p f_{X X} g\right\} d \alpha} .\end{array}\right.$

(ii) $X$ is non observed and hence it must be privately optimal for the farmer given the set of contracts $(Z(\alpha), T(\alpha))$

$$
\int_{\underline{\alpha}}^{\bar{\alpha}} U^{\prime}(\Pi(\alpha)) p f_{X}(\alpha, X, Z(\alpha)) d G(\alpha)=0,
$$

(iii) a strictly positive informational rent is left to any farmer with type $\alpha>\underline{\alpha}$ :

$$
\Pi(\alpha)=\Pi(\underline{\alpha})+\int_{\underline{\alpha}}^{\alpha} p f_{\alpha}(u, X, Z(u)) d u .
$$

(iv) If $U$ is $C A R A$, then the less efficient farmer has no rent $(\Pi(\underline{\alpha})=0)$. Otherwise, the less efficient farmer may earn a strictly positive rent $(\Pi(\underline{\alpha})>0)$.

Proof: Available from the authors upon request

The conditions described in Proposition 1 together determine the optimal solution of the agency's program concerning the optimal nitrogen quota $Z($.$) , the optimal level of rents$ 
left to farmers $\Pi($.$) and the optimal first application X$. Conditions (ii) simply recall that the optimal choice of first nitrogen application $X$ should maximize the expected utility of farmer's profit (moral hazard constraint). Condition (iii) states the level of informational rent left to any type- $\alpha$ farmer. All farmers may earn strictly positive rents at the optimum. Intuitively, this may happen because of the presence of the certainty equivalent of profit in welfare. In order to maximize certainty equivalent or equivalently to minimize the associated risk premium, the regulator may find it optimal to leave rents even to the most inefficient type of farmers $(\underline{\alpha})$, contrary to what happens in most adverse selection models.

Moreover, compared to the perfect information case described in (3), two new terms should be added in the marginal cost determining the nitrogen quota $Z$ (see condition (i)). This is because the regulator has to take into account two sets of constraints due to the unobservability of both the first application $X$ and parameter $\alpha$. Let us rewrite (i) by omitting arguments for simplicity:

$$
p f_{Z}=w-\frac{\kappa}{(1+\lambda)} U^{\prime}(\Pi) p f_{X Z}-\frac{\nu}{(1+\lambda) g} p f_{\alpha Z}
$$

The marginal private benefit of $Z$ should be equalized with the virtual marginal cost of $Z$, defined by the right hand side of (5). This virtual marginal cost is the sum of the unit cost $(w)$, the marginal cost of incentives due to moral hazard on $X\left(-\kappa U^{\prime}(\Pi) p f_{X Z} /(1+\lambda)\right)$, and the marginal cost of incentives due to incomplete information on $\alpha\left(-\nu p f_{\alpha Z} /((1+\lambda) g)\right)$.

The direction of both distortions depend on the particular specification of the model. In particular these distortions will depend on the sign of shadow prices $\nu(\alpha)$ and $\kappa$. To better understand the source of those incentive distortions in our framework which incorporates simultaneously moral hazard, incomplete information and risk sharing problems, it is useful to consider these in turn.

First, let us concentrate on the incomplete information problem regarding $\alpha$. For this, consider that the farmer is risk-neutral $\left(U^{\prime}(\Pi)=1\right)$ and assume further that $X$ is observable so that the moral hazard problem disappears. In this case without risk aversion and moral 
hazard, our model is similar to a standard adverse selection model (e.g. Baron and Myerson). Hence the shadow price $\kappa$ of the moral hazard constraint is 0 and we obtain easily that condition (i) rewrites simply as:

$$
p f_{Z}=w-\frac{\nu^{B M}}{(1+\lambda) g} p f_{\alpha Z}
$$

where $\nu^{B M}(\alpha)=-\lambda(1-G(\alpha)) \leq 0$ and where $B M$ stands for Baron-Myerson. Because $f_{\alpha Z}<0$ and $\nu^{B M}(\alpha) \leq 0$, incomplete information on $\alpha$ leads the principal to distort upwards the level of $Z(\alpha)$ for any $\alpha$ except for the most efficient $(\bar{\alpha})$. The intuition of the result proceeds as follows. Note that, from condition (iii), the rate of growth in farmer's rent is still $\Pi^{\prime}(\alpha)=p f_{\alpha}$ and that $Z(\alpha)$ for the type- $\alpha$ farmer has an impact only on rents of all more efficient farmers. Intuitively, by increasing the allowed total nitrogen quota $Z$ over the perfect information level, the agency is able to decrease $\Pi^{\prime}(\alpha)$ as $f_{\alpha Z}<0$ and consequently to extract more socially costly rents from all more efficient farmers than $\alpha$, in a proportion $(1-G(\alpha))$. From this perspective, we recover the classic result of adverse selection models which stipulates that rent extraction by the regulator comes at a social cost, i.e., by distorting upwards total nitrogen level in our case.

Second, let us go one step further by introducing risk aversion for the agent while still assuming that $\alpha$ is private information to the farmer and that $X$ is observable. In that situation which combines both hidden information and risk sharing as in Laffont and Rochet, condition (i) rewrites as follows:

$$
p f_{Z}=w-\frac{\nu^{L R}}{(1+\lambda) g} p f_{\alpha Z}
$$

where $\nu^{L R}(\alpha)=\nu^{B M}(\alpha)+\int_{\alpha}^{\bar{\alpha}}\left(\frac{U^{\prime}(\Pi(u))}{U^{\prime}(C E(\Pi))}-1\right) g(u) d u$ and where $L R$ stands for LaffontRochet. The regulator has now to worry about the impact of rent extraction through distorting $Z$ on the certainty equivalent of profit $C E(\Pi)$ which is part of the regulatory objective. Note first that risk aversion does not modify the previous result of no distortion at the top because we still have that $\nu^{L R}(\bar{\alpha})=\nu^{B M}(\bar{\alpha})=0$. Moreover, the direction of the distortion due to incentives is determined by the sign of $\nu^{L R}(\alpha)$. It is possible to show that when $U$ is 
CARA or IARA then $\nu^{L R}(\alpha) \leq \nu^{B M}(\alpha) \leq 0$, so that risk aversion for the farmer tends to increase the cost of incentive compatibility and hence the size of the downward distortion. In the DARA case, there exists a threshold type $\tilde{\alpha}$ such that for any type less (resp. more) efficient, i.e., $\alpha \leq(\geq) \tilde{\alpha}$, the cost of incentive compatibility tends to be lower (greater) than under risk neutrality $\left(\nu^{L R}(\alpha) \geq(\leq) \nu^{B M}(\alpha)\right)$ (see Appendix 3 for a formal statement).

Finally, by considering further that $X$ is non observable for the regulator and hence non contractible, our framework can be seen as a generalization of Laffont-Rochet. In our model, moral hazard, hidden information and risk sharing problems do not simply add. First of all, one must add a new source of distortion on $Z$ given by the term $-\frac{\kappa}{(1+\lambda)} U^{\prime}(\Pi) p f_{X Z}$ in $(5)$. This term indicates that each change in $Z(\alpha)$ for the type- $\alpha$ farmer leads the regulator to take into account the induced effect on the social cost of the moral hazard constraint. Indeed, as $X$ and $Z$ are complements, any increase in $Z$ also increases the marginal productivity of $X$ and so the social cost of moral hazard. This effect can be interpreted as the direct effect from the presence of moral hazard on the cost of hidden information. The direction of the corresponding distortion on $Z$ is driven by the sign of the shadow price of moral hazard: If $\kappa<0$, which means that the farmer overuses $X$ from the regulator's perspective, then it is optimal to distort $Z$ downwards $\left(-\frac{\kappa}{(1+\lambda)} U^{\prime}(\Pi) p f_{X Z}>0\right)$ in order to induce a lower level of $X$. On the contrary, when there is underprovision of $X$ at the optimum $(\kappa>0)$, then the regulator distorts $Z$ upwards. Secondly, in condition (i) the last term in $\nu(\alpha)$, i.e. $\kappa \int_{\alpha}^{\bar{\alpha}}\left\{p f_{X}(u, X, Z(u)) U^{\prime \prime}(\Pi(u))\right\} g(u) d u$, appears only if the regulator faces simultaneously moral hazard, risk sharing and hidden information problems. This term states that whenever the regulator manipulates the quota $Z(\alpha)$ for the type- $\alpha$ farmer, then he will take into account the induced effect on the rent $\Pi(\alpha)$ of all more inefficient farmers through condition (iii) and that this in turn will impact the social cost of moral hazard constraint through condition (ii). This effect can be interpreted as an indirect effect from the presence of moral hazard and risk aversion on the cost of hidden information. The sign of this term is generally ambiguous because $f_{X}$ is not of constant sign. 
Importantly, in our setting, there is always a distortion at the top (i.e., for $\alpha=\bar{\alpha}$ ), even if the farmer is risk neutral $\left(U^{\prime}()=1.\right)$. Indeed, in that case, the condition on $Z$ in (i) rewrites

$$
p f_{Z}=w-\frac{\kappa}{(1+\lambda)} p f_{X Z}-\frac{\nu}{(1+\lambda) g} p f_{\alpha Z}
$$

with $\nu(\alpha)=\nu^{B M}(\alpha)=-\lambda\left(1-G(\alpha)\right.$ and $\kappa=-\left(\int_{\underline{\alpha}}^{\bar{\alpha}}\left\{S_{X}-(\lambda(1-G(\alpha))) p f_{\alpha X}\right\} d \alpha\right) /\left(\int_{\underline{\alpha}}^{\bar{\alpha}}\left\{p f_{X X} g\right\} d \alpha\right)$.

At the top, we have $\nu(\bar{\alpha})=0$ but the direct effect due to the moral hazard constraint still remains.

\section{Empirical application}

We deal in this section with the calibration of the theoretical model described above, using production function estimates and specifying the regulator and the agent preferences. Even though our model is entirely virtual, it belongs to a category of contract-based economic instruments for environmental regulation that may well be used in practice. Hence, realworld data on agricultural production are necessary to provide a more realistic framework for policy evaluation.

We first present the data set used and provide some basic statistics for crop yield and nitrogen fertilizer use. We then report estimation results of the crop yield function depending on nitrogen fertilizer applications at different stages of the production process. Estimation results are then used to calibrate and simulate the optimal contract by specifying the distribution of $\alpha$, the farmer utility function and the environmental damage function.

\section{Data presentation}

We used data from the "Cropping Practices" survey conducted in 1994 by the French Ministry of Agriculture (SCEES). A representative population of farmers is first selected from a nationwide sample of cereal farmers. For this population, a subset of land plots by major crop is chosen, so that the new population of plots is also representative in terms of land acreage. 1844 plots are retained for corn production in 1994. For each land plot, a very detailed 
description of the crop production process is available: land preparation, chemical and organic fertilizer applications, pesticide use, etc. This data set is particularly adapted to our empirical framework, as the fertilizer application stages are also recorded before, during and after seeding. As the sample consists of land plots located in various regions of the country, unobserved heterogeneity of plots due to climatic conditions and soil quality is very likely. To control partly for this heterogeneity, we selected land plots for which no organic fertilizer application has been made.

A major problem in our dataset is the lack of information on nitrogen carry-over from previous crops. The only information available is the nature of the previous crop, and the proportion of crop residues buried in the soil. After removing observations with missing variables on either first- or second-period nitrogen fertilizer applications, we are left with a complete sample of 341 observations. Table 1 presents descriptive statistics on the sample.

\section{Model estimation and calibration}

The production function is $R=F(\tilde{X}, \tilde{Y})$, where $\tilde{X}=\alpha \gamma(A+X), \tilde{Y}=\alpha \gamma(1-\gamma)(A+X)+\gamma Y$ and $Y=Z-X$, or, in terms of first-period $(X)$ and second-period $(Y)$ fertilizer applications, $R=\hat{f}(A, \alpha, \gamma, X, Y)$ where $A, \alpha$ and $\gamma$ are unknown parameters. Assuming the "true" yield function is quadratic in $\tilde{X}$ and $\tilde{Y}$, an equivalent reduced form can be written as a quadratic form of $X, Y$, by replacing $\tilde{X}$ and $\tilde{Y}$ by their expressions:

$$
R=b_{0}+b_{1} X+b_{2} Y+b_{3} X^{2}+b_{4} Y^{2}+b_{5} X Y \text {. }
$$

A particular attention has to be paid to endogeneity of explanatory variables in the model above. There are two possible sources of endogeneity which may yield inconsistent parameter estimates if an ordinary least squares procedure is used. First, due to the sequential nature of the economic model, the second-period fertilizer application $Y$ is an implicit function of $X$. Hence, the conditional expectation of crop yield $R$ given $X$ captures not only the "direct effect" on yield of the first-period application, but also the "indirect effect" of $X$ through 
the level of $Y$. Second, both $X$ and $Y$ may be correlated with unobserved heterogeneity in crop yield, e.g., climatic conditions and soil quality may affect farmer decisions on nitrogen fertilizer applications, while having a direct impact on yield. For these reasons, it is necessary to use an instrumental variable estimation technique for producing consistent reduced-form parameter estimates. Note however that, while $Y$ is very likely to be endogenous in the sense described above, $X$ may be exogenous, i.e., it may be uncorrelated with the error term in the crop yield function. However, the cross-application variable $X \times Y$ would not be exogenous if $Y$ is endogenous. The equation above is estimated by the instrumental variable (Two-stage Least Squares) method, using as instruments AVTEMP, AVRAIN, AVWIND (respectively, average temperature, precipitation and wind velocity over the growing season), PLOUGH (dummy, 1 if the land plot was ploughed before planting), and SETASIDE (dummy, 1 if the land plot was left idle during the previous growing season).

The augmented-regression test procedure (Davidson and MacKinnon) is used to check for the exogeneity of $X$ and $X^{2}$ in the equation. The computed Wald test having value $\chi^{2}(3)=3.25$ with a p-value of 0.3545 , we fail to reject the null hypothesis of exogeneity of $X$ and $X^{2}$. We also perform the same test for the validity of the whole set of instruments. The Wald test in this case has a p-value of 0.7466 , allowing us to conclude that instrumental variable estimates are consistent.

There are six reduced-form parameters $b_{j}, j=0,1, \ldots, 5$ and three incidental parameters $A, \alpha, \gamma$. To identify the structural model above from first-order moments only, it is necessary to add three parameter restrictions. From the instrumental variable estimation, it appears that the restriction $b_{5}=3 b_{3}$ is not rejected at the five percent level (the associated Fisher test statistic has a p-value of 0.3145$)$. Two additional restrictions are obtained by setting $A=35$ $\mathrm{kg} /$ ha and $\gamma=0.975$; these values are such that the first-order condition $\partial E \Pi(Z, X) / \partial X$ is as close as possible to 0 , when $\alpha$ is set to $0.5 .{ }^{9}$ Estimation results for the reduced-form model are presented in table $2 .^{10}$

Parameter $\alpha$ is distributed in the domain $[0,1]$, and its distribution could be left- or 
right-skewed. The probability distribution function for $\alpha$ is specified as a Beta distribution $B\left(z_{1}, z_{2}\right)$, where $z_{1}=z_{2}=1.4$, so that $\alpha$ has mean 0.50 and a standard deviation of 0.125 .

In our simulation experiment, we do not account for variations in surplus that may originate from the agricultural-good market, in order to concentrate on the environmental issue. Indeed, as we assumed earlier that output price is constant, the variation of consumer surplus comes only from variations in environmental damage. We specify the environmental damage function, i.e., $S=-D$, where $D(\alpha, X)=\eta[(1-\alpha)(A+X)]^{2}$. Setting $\eta=0.5$ together with all parameters choices allows us to obtain average values for the nitrogen level $Z$ close to its sample mean when there is no public intervention and for $\rho=4 .^{11}$

\section{Results and comments}

The attitude toward risk for farmers is likely to be an important element that will condition the shape of the optimal solution. More precisely, a comparison between the risk-neutrality and the risk-aversion cases will allow us to discuss the inherent regulation cost related to risk hedging for farmers. The risk-neutrality case can then be used as a benchmark, to be compared with increasing values for the risk aversion parameter.

We will consider several cases in the simulation experiment: Perfect Information, Risk neutrality $(U(\Pi)=\Pi)$ and CRRA (Constant Relative Risk Aversion) utility with $U(\Pi)=$ $\Pi^{1-\rho} /(1-\rho)$. The choice of a CRRA utility function is motivated on empirical grounds (Moschini and Hennessy). Moreover, we also present the private equilibrium solution (denoted status quo), which is obtained by solving (1) with $T=0$ (table 3 ).

In the status quo case, our simulation results indicate that both the optimal level of first nitrogen application and the expected total nitrogen application are decreasing in the risk aversion parameter $\rho$. Thus, nitrogen application is globally risk increasing, a result previously obtained, as noted above, by Hennessy and Roosen for U.S. corn producers under the same assumption of non-conditional distribution of uncertainty. As a consequence, total surplus is positive when the risk aversion parameter is sufficiently large ${ }^{12}$ (e.g., for $\rho=4$ or 
5), but it is negative for lower values because the environmental damage is large. In any case, potential emissions from initial nitrogen stock $X$ are not internalized by the farmer.

Based on the estimation results above, we are now in a position to simulate the optimal contract described in the theoretical part of the article. Because of the complexity of the optimal solution, it is necessary to rely on numerical techniques to construct optimal paths of variables of interest, as analytical solutions are not available (see the appendix for more details on the numerical procedure).

We present in table 4 the total surplus, with three components (environmental damage, transfer, certainty-equivalent of profit), and the solution for $X$ for the perfect information, risk neutrality and CRRA cases. For CRRA, the certainty equivalent first increases and then decreases with $\rho$ (it is equal to expected profit in the risk neutrality case), and is equal to 0 in the perfect information case. The trend in initial nitrogen $X$ is clearly increasing with risk aversion, with values much greater than for the perfect information case. Also, it is interesting to note that under regulation, initial nitrogen application is lower than in the status quo case, except when $\rho$ is large (e.g., for $\rho=5$ ). Overall, total surplus computations indicate that public intervention is not profitable when $\rho=4$ or 5 , as total surplus under regulation becomes lower than under status quo. In this case, the cost of asymmetric information and moral hazard is so high that laissez-faire is better than public intervention.

Figure 2 presents optimal nitrogen paths $Z(\alpha)$ for selected values for $\rho$, where we report the status quo solution under risk neutrality only. Status quo and perfect information nitrogen paths do decrease over the interval $[\underline{\alpha}, \bar{\alpha}]$. Regulation under perfect information entails a higher level of total nitrogen use for every farmer (except for some very efficient, i.e. $\alpha$ greater than 0.9) that compensates them for a mandatory reduction in $X$, compared to the status quo. Turning now to the risk neutrality case under imperfect information, information asymmetry implies a lower level of total nitrogen over the entire range of $\alpha$ compared to the two cases above. In the risk-neutrality case, due to the cost of hidden information and moral hazard, the regulator is induced to reduce drastically the level $Z$ so that the constraint 
$Z \geq X$ is binding for all values of $\alpha$. Also, risk-neutral farmers are partly compensated by a level of $X$ greater than in the perfect information case. To sum up, with risk neutrality, distortions due to asymmetric information are such that the optimal policy is uniform for all farmers: a second application of nitrogen $Y$ is not allowed and all farmers are induced to use a level $X=39.06 \mathrm{~kg} / \mathrm{ha}$ and are compensated via a constant transfer $T$. The optimal policy provides no flexibility with respect to adjustment of nitrogen once $\alpha$ is learned.

In the CRRA case $(\rho>0)$, the constraint $Z \geq X$ is binding only for interior values of $\alpha$ and the cut-off point where $Z(\alpha)=X, \forall \alpha$, is monotonically increasing with $\rho$. Also $X$ is increasing with the risk aversion parameter (table 4). Optimal nitrogen paths start from higher values (at $\alpha=\underline{\alpha}$ ) when risk aversion is greater. The monotonic feature of the cut-off point in nitrogen paths as $\rho$ increases originates from distortions depending on the sign of $\nu(\alpha), \kappa$ recalling that $f_{X Z}>0$ and $f_{\alpha Z}<0$. Indeed, recall that the equation determining the optimal level $Z$ is:

$$
p f_{Z}=w-\frac{\kappa U^{\prime}(\Pi) p f_{X Z}}{1+\lambda}-\frac{\nu p f_{\alpha Z}}{(1+\lambda) g(\alpha)}
$$

Thus, marginal profit of $Z\left(p f_{Z}\right)$ is equal to unit price $w$ less two terms, the first one corresponding to the moral hazard constraint and the second one to the incentive compatibility constraint. Actually, both distortions are positive so that moral hazard and incentive compatibility constraints entail a downward distortion on $Z$ whatever $\alpha$, compared to the perfect information case. Then, for the chosen values of $\rho$ and $\alpha$, the optimal nitrogen level is always lower than the perfect information level. However, this means that the constraint $Z \geq X$ also binds for the most efficient types. In the simulation experiment, the value of $\kappa$ is always negative, which is consistent with the idea that farmers tend to overuse nitrogen fertilizer from the regulator's perspective. As discussed above, it is therefore optimal to distort $Z$ downwards, in order to induce a reduction in $X$.

Moreover, figure 2 indicates that the range $Z(\bar{\alpha})-Z(\underline{\alpha})$ is much more restricted for low values of $\rho$, e.g., $\rho=0$ or $\rho=2$, compared to the range for highest values of this 
parameter, e.g., $\rho=4$ or $\rho=5$. Note finally that, contrary to usual models of regulation under asymmetric information, there is a distortion "at the top", which means here that even the type $\bar{\alpha}$ underproduces at the optimum. Indeed, even though $\nu(\bar{\alpha})=0$, moral hazard still introduces a distortion through the second term in the right-hand side of equation (8) above.

Figure 3 confirms that profits are increasing with $\alpha$ in all cases (profit in the perfect information case is equal to 0 , as the regulator is able to set transfer so that farmer profit is equal to her reservation level). Profit path under regulation starts from $\Pi(\underline{\alpha})=0$ under risk neutrality and rent extraction is highest compared to what happens when $\rho>0$. When $\rho$ increases, it is optimal to increase $\Pi(\underline{\alpha})$ in order to maximize the certainty equivalent of profit or equivalently to reduce the associated risk premium (for $\rho$ from 0.5 to $5, \Pi(\underline{\alpha})$ is equal to 91.01 Euros to 95.43 Euros). Hence, for $\rho \in\{0.5,2,3,4,5\}$, profit paths start from 91.01 approximately to values between 426.85 Euros/ha and 503.08 Euros/ha. Interestingly, the potential for rent extraction does not vary monotonically with the degree of risk aversion.

From tables 3 and 4, the marginal benefits from environmental regulation can easily be calculated, in terms of nitrogen and damage reduction. For example, when $\rho=0$ (risk neutrality), $X$ decreases by 73 percent compared to the status quo, and damage by 83 percent, for a unit transfer of 93.71 Euros/ha. Therefore, the cost of the program is around 0.88 Euros for each $\mathrm{kg}$ of nitrogen abated, whereas the marginal value of damage reduction per Euro of subsidy $(\Delta D /(1+\lambda T))$ equals 6.89 . When $\rho=2, X$ is reduced by 55 percent and damage by 51 percent compared to the status quo, and the cost of the program is about 0.98 Euros for each $\mathrm{kg}$ of nitrogen abated, with a marginal damage reduction per Euro of subsidy around 6.40. In the case of a negative transfer (a tax), when $\rho=3$, the program achieves a marginal value of nitrogen reduction of 0.13 Euros for each $\mathrm{kg}$ of nitrogen abated, and a marginal value of damage reduction of 47.32 per Euro of tax.

Finally, figure 4 depicts transfers as a function of the quota $Z$. This is equivalent to non linear pricing of nitrogen. One can see that risk aversion has also a large impact on the nature of transfer $T(Z)$. While all types of farmer are taxed when information is perfect, asymmetric 
information entails that, as soon as the level of $Z$ is sufficiently high (or equivalently, when $\alpha$ is sufficiently low), the farmer is subsidized at a rate increasing with the level of nitrogen used. This is because the regulator is highly constrained by the fact that every farmer should earn a non negative ex post rent. In fact, it could be optimal for the regulator to exclude the worst types of farmers (i.e., farmers with $\alpha$ close to $\underline{\alpha}$ ) by granting them a lumpsum transfer independent from $\alpha$, for ceasing production. ${ }^{13}$

\section{Conclusion}

In this article, we have analyzed a model of contract-based regulation for a risk-averse farmer, involving moral hazard, hidden information and risk sharing problems. The farmer faces a production risk because of nitrogen leaching, and he privately observes the soil capacity in retaining nitrogen after the contract is signed. The optimal contract policy specifies a quota for nitrogen input use, whose decomposition over different production periods is unknown to the regulator.

The optimal solution has been characterized under general assumptions on net expected social surplus and the farmer utility function. Social surplus includes an environmental damage function related to nitrogen leaching. To produce more realistic insights in terms of environmental performance of this virtual contract policy, the farmer's sequential decision model is estimated on French crop production data, and the results are used to calibrate and simulate the optimal contract.

We show that, compared to the perfect information case, two distortions should be added to the condition determining the screening variable of the model (i.e., in our case the level of total nitrogen $Z$ ). This is because the regulator has to take into account two sets of constraints due to the unobservability of both the first nitrogen application $X$ and soil nitrogen retention index $\alpha$. The directions of both distortions depend on the particular specification of the model. Risk aversion is shown to have a large impact on the pattern of the optimal regulation, implying that the farmer may be subsidized at the equilibrium, whereas he is taxed under 
perfect information. Moreover, in our empirical application, there is underproduction at the top compared to the perfect information outcome, and this is true even if the farmer is risk neutral. When the coefficient of risk aversion becomes large (more than 4 in our application), total surplus obtained under regulation is in fact less than the status quo one, and eventually the environmental damage becomes larger. Hence, public intervention should not be recommended in that case. Finally, all farmers may earn strictly positive rents at the optimum.

Having a look at figures 2 and 3, one can easily see that if risk preferences are unknown to the regulator, e.g., if the particular value of risk parameter $\rho$ is an adverse selection parameter, then the contracts analyzed here are not incentive compatible with respect to risk preferences. This means that the design of contract should take into account the possibility of strategic behavior by the farmer regarding the revelation of her risk preferences. The task of finding an optimal mechanism in this situation involving multi-dimensional hidden information is devoted to future research. 


\section{Appendix}

\section{Appendix 1}

We denote $F_{1}=\partial F / \partial \tilde{X}, F_{2}=\partial F / \partial \tilde{Y}$ and $F_{12}=\partial^{2} F / \partial \tilde{Y} \partial \tilde{X}$ and we have:

$$
\frac{\partial f}{\partial \alpha}=F_{1} \frac{\partial \tilde{X}}{\partial \alpha}+F_{2} \frac{\partial \tilde{Y}}{\partial \alpha}=\gamma(A+X)\left(F_{1}+(1-\gamma) F_{2}\right)>0
$$

and

$$
\frac{\partial^{2} f}{\partial \alpha \partial Z}=\gamma^{2}(A+X)\left(F_{12}+(1-\gamma) F_{22}\right)<0
$$

Recall that we have assumed $F_{12}<0$, so that both first and second nitrogen up-take are substitutes. Together with the concavity of $F$, this clearly implies that $\frac{\partial^{2} f}{\partial \alpha \partial Z}<0$. Moreover, $\frac{\partial f}{\partial Z}=F_{2} \frac{\partial \tilde{Y}}{\partial Z}=\gamma F_{2}>0$ and $\frac{\partial^{2} f}{\partial Z^{2}}=\gamma^{2} F_{22} \leq 0$. Finally, we have:

$$
\frac{\partial f}{\partial X}=F_{1} \frac{\partial \tilde{X}}{\partial X}+F_{2} \frac{\partial \tilde{Y}}{\partial X}=\alpha \gamma F_{1}+[\alpha \gamma(1-\gamma)-\gamma] F_{2}
$$

and

$$
\frac{\partial^{2} f}{\partial X \partial Z}=\alpha \gamma^{2} F_{12}+\gamma[\alpha \gamma(1-\gamma)-\gamma] F_{22}
$$

whose sign is a priori ambiguous because the common term between brackets is negative for any $\alpha$ and $\gamma$ that belong to $[0,1]$.

\section{Appendix 2}

We wish here to exhibit conditions under which the risk averse farmer reduces the first application level $X$ compared to a risk neutral one. In the absence of any regulation, the program of the farmer with respect to $X$ is

$$
\max _{X} \int\left[U\left(\Pi\left(\alpha, X, Z^{\circ}(\alpha, X)\right)\right)\right] d G(\alpha) \quad \text { s.t. } \quad 0 \leq X \leq Z^{\circ}(\alpha, X),
$$

and the corresponding first-order condition (for an interior solution) is

$$
\int\left[U^{\prime}\left(\Pi\left(\alpha, X, Z^{\circ}(\alpha, X)\right)\right) p f_{X}\left(\alpha, X, Z^{\circ}(\alpha, X)\right)\right] d G(\alpha)=0
$$


or equivalently

$$
\operatorname{Cov}\left(U^{\prime}, p f_{X}\right)+E\left(U^{\prime}\right) E\left(p f_{X}\right)=0
$$

For the risk neutral farmer, this condition reduces simply to $E\left(p f_{X}\left(\alpha, X^{r n}, Z^{\circ}\left(\alpha, X^{r n}\right)\right)\right)=0$ and defines $X^{r n}$ as the optimal first application, where $r n$ stands for risk-neutral.

Let us denote $X^{r a}$ the optimal solution for the risk averse farmer. The risk averse farmer reduces $X$ if and only if $E\left(p f_{X}\left(\alpha, X^{r a}, Z^{\circ}\left(\alpha, X^{r a}\right)\right)\right)>0$. Recall that $\Pi$ is increasing in $\alpha$ so that $U^{\prime}(\Pi)$ is decreasing in $\alpha$. Also, differentiating totally $f_{X}$ w.r.t. $\alpha$, we get

$$
\frac{d f_{X}}{d \alpha}=f_{\alpha X}+f_{X Z} \frac{\partial Z^{\circ}}{\partial \alpha}
$$

A necessary and sufficient condition for $E\left(p f_{X}\right)>0$ is then $\frac{d f_{X}}{d \alpha}>0$. Because $f_{X Z}>0$ and $\frac{\partial Z^{\circ}}{\partial \alpha}=-\frac{f_{\alpha Z}}{f_{Z Z}}<0$ (recall that $Z^{\circ}$ is given by $p f_{Z}=w$ and that $f_{Z Z}<0, f_{\alpha Z}<0$ ), this condition is equivalent to

$$
f_{\alpha X} f_{Z Z}-f_{X Z} f_{\alpha Z}<0
$$

Appendix 3

We have

$$
\begin{aligned}
\nu^{L R}(\alpha)-\nu^{B M}(\alpha) & =\int_{\alpha}^{\bar{\alpha}}\left(\frac{U^{\prime}(\Pi(u))}{U^{\prime}(C E(\Pi))}-1\right) g(u) d u \\
& =\int_{\alpha}^{\bar{\alpha}}\left(\frac{U^{\prime}(\Pi(u))}{U^{\prime}(C E(\Pi))}\right) g(u) d u-(1-G(\alpha)) .
\end{aligned}
$$

Let us denote $\phi(\alpha)=\int_{\alpha}^{\bar{\alpha}} U^{\prime}(\Pi(u)) g(u) d u-(1-G(\alpha)) U^{\prime}(C E(\Pi))$. We have $\phi(\bar{\alpha})=0$ and

$$
\phi^{\prime}(\alpha)=\left[U^{\prime}(C E(\Pi))-U^{\prime}(\Pi(\alpha))\right] g(\alpha) .
$$

As $U^{\prime}(\Pi(\alpha))$ is decreasing in $\alpha, \phi^{\prime}(\alpha)$ is first negative and then positive. Hence if $\phi(\underline{\alpha}) \leq 0$, then $\nu^{L R}(\alpha) \leq \nu^{B M}(\alpha) \leq 0$ for any $\alpha$. Note that $\phi(\underline{\alpha}) \leq 0$ is equivalent to $\int_{\underline{\alpha}}^{\bar{\alpha}} U^{\prime}(\Pi(u)) g(u) d u-$ $U^{\prime}(C E(\Pi))<0$ which in turn is equivalent to saying that the function $U^{\prime}\left(U^{-1}().\right)$ is concave, according to the Jensen inequality. Moreover $\frac{d}{d \Pi}\left[U^{\prime}\left(U^{-1}(\Pi)\right)\right]=-\rho(\Pi)$ where $\rho()=.-\frac{U^{\prime \prime}(.)}{U^{\prime}(.)}$ is the Arrow-Pratt measure of local risk aversion. Hence we have that $\phi(\underline{\alpha}) \leq 0$ is finally equivalent to $U($.$) being CARA or IARA. Similarly, \phi(\underline{\alpha})>0$ is equivalent to $U($.$) being$ 
DARA. Consequently, in the DARA case, there exists a threshold type $\tilde{\alpha}$ such that for any $\alpha \leq \tilde{\alpha}$ we have $\nu^{L R}(\alpha) \geq \nu^{B M}(\alpha)$ and for any type $\alpha \geq \tilde{\alpha}$ we have $\nu^{L R}(\alpha) \leq \nu^{B M}(\alpha)$. Appendix 4

First, we reduce the complexity of incentive constraints. As it is usual in adverse selection models (see Guesnerie and Laffont), (IC) can be reduced to the following set of conditions

$$
\begin{gathered}
\dot{\Pi}=p f_{\alpha}(\alpha, X, Z(\alpha)), \\
f_{\alpha Z} \dot{Z} \geq 0 .
\end{gathered}
$$

Recall that $f$ is increasing in $\alpha$ and that $f_{\alpha Z}=\partial^{2} f / \partial \alpha \partial Z$ is strictly negative. The latter property of $f$ corresponds to the usual single crossing condition that simplifies greatly the analysis of optimal contracts (Guesnerie and Laffont). Given that $\dot{\Pi}$ is strictly positive as indicated by (9), constraints (IR) reduce to:

$$
\Pi(\underline{\alpha}) \geq 0
$$

Hence it suffices to check for the participation constraint of the less efficient farmer. Moreover, as $f_{\alpha Z}<0$, (10) reduces to $\dot{Z} \leq 0$. The optimal quota is thus non-increasing in $\alpha$.

We now define $\dot{Z}=-\psi$, where $\psi$ is a control variable and we redefine $Z$ as a state variable. Then, constraint (10) reads as $\psi \geq 0$. Moreover, we redefine $X$ as a state variable with the equation $\dot{X}=0$ and $X(\alpha) \geq 0, X(\underline{\alpha})=X, X(\bar{\alpha})$ free. Second, we use a first order approach for the moral hazard constraint on $X{ }^{14}$ Hence, the first order condition corresponding to constraint $(\mathrm{MH})$ is

$$
\int_{\underline{\alpha}}^{\bar{\alpha}}\left[\frac{\partial U(\Pi(\alpha))}{\partial X}\right] d G(\alpha)=\int_{\underline{\alpha}}^{\bar{\alpha}} M(\alpha, X(\alpha), Z(\alpha), \Pi(\alpha)) d G(\alpha)=0,
$$

where $M=U^{\prime}(\Pi(\alpha)) p f_{X}(\alpha, X(\alpha), Z(\alpha))$. To deal with this integral constraint, we define a new state variable

$$
\dot{K}=M(X(\alpha), Z(\alpha), \Pi(\alpha), \alpha) g(\alpha) \quad \text { with } K(\underline{\alpha})=K(\bar{\alpha})=0
$$


and let $\mathcal{W}(\alpha, X(\alpha), Z(\alpha), \Pi(\alpha))$ denote the term equal to:

$$
\begin{aligned}
\mathcal{W}(\alpha, X(\alpha), Z(\alpha), \Pi(\alpha))= & S(\alpha, X(\alpha), Z(\alpha))+(1+\lambda) p f(\alpha, X(\alpha), Z(\alpha)) \\
& -(1+\lambda)(\Pi(\alpha)+w Z(\alpha)) .
\end{aligned}
$$

We then transform the program (P1) into an optimal control problem:

$$
\max _{\psi} C E(\Pi)+\int_{\underline{\alpha}}^{\bar{\alpha}} \mathcal{W}(\alpha, X(\alpha), Z(\alpha), \Pi(\alpha)) d G(\alpha),
$$

subject to

$$
\begin{aligned}
& \dot{X}=0, \dot{\Pi}=p f_{\alpha}(\alpha, X(\alpha), Z(\alpha)), \dot{Z}=-\psi, \dot{K}=M(\alpha, X(\alpha), Z(\alpha), \Pi(\alpha)) g(\alpha), \\
& Z(\alpha)-X(\alpha) \geq 0, \psi(\alpha) \geq 0, X(\alpha) \geq 0, \\
& X(\underline{\alpha})=X, \Pi(\underline{\alpha}) \geq 0, K(\underline{\alpha})=K(\bar{\alpha})=0,
\end{aligned}
$$

with one control variable $(\psi)$ and four state variables ( $\Pi, X, Z, K)$.

Appendix 5. Numerical simulation procedure

We describe here the numerical procedure used to solve for the optimal solution. We first generate 100 equidistant values for $\alpha$ in the interval $[0,1]$, and choose initial values for quota $Z$ as $z_{0}=380(1-\alpha)$, and an initial value for $X, X_{0}=65$.

For a given value of the risk-aversion coefficient, we start a loop over all values of $\alpha$, and compute $\kappa$ and $\nu$ given in Proposition 1 for each $\alpha$. This part of the numerical algorithm is particularly computationally demanding, specially for parameter $\kappa$ as it involves a ratio of (multiple) integrals. Integrals are approximated by discretization over the $[0,1]$ interval, with a step of 0.01 .

We then solve for $X$ in condition (ii) of Proposition 1, using a numerical root-finding algorithm. The next step is to compute the next value of the quota $Z(\alpha)$ using condition (i) in the Proposition. After new values for $X$ and individual quotas $Z(s)$ for $s=0, \ldots, \alpha$ have been found, we compute profit $\Pi$ from condition (iii). We use the last result in the appendix to compute $\Pi(\underline{\alpha})$.

We then replace initial values $X_{0}$ and $Z_{0}$ with new values $X_{n e w}, Z_{n e w}$, using a smoothing parameter 0.6: $Z(\alpha)_{0}=0.6 Z(\alpha)_{n e w}+0.4 Z(\alpha)_{0}$ for $\alpha \in[0,1]$, and $X_{0}=0.6 X_{n e w}+0.4 X_{0}$. This 
smoothing technique allows for soft convergence as the algorithm might otherwise become stuck if successive values for $Z$ are too far apart. The final step in the algorithm is to test for convergence in $X$ and the $Z$ and $\Pi$ functions, with a criterion of $10^{-8}$ for average relative variations (in percent). 


\title{
Notes
}

\begin{abstract}
${ }^{1}$ Indeed, it would then be possible for the principal to design a contract inducing the first best outcome where the agent supports all the risk and thus takes all appropriate decisions that maximize principal's gain. This contract also provides the agent exactly with her reservation utility in expectation.
\end{abstract}

${ }^{2}$ Note that we assume that unit price is the same for $X$ and $Y$, although there is no loss of generality associated with this assumption. We also assume that the nitrogen price $w$ is constant over the whole period.

${ }^{3}$ See Appendix 1 for details.

${ }^{4}$ This means in particular that we assume prices $p$ and $w$ to be non random.

${ }^{5}$ Another important assumption in the model is the independence of the probability distribution function from $X: G(\alpha \mid X)=G(\alpha) \forall \alpha$. With this assumption, the riskiness in the production process cannot be controlled by adjusting the level of $X$ in the first stage. This differs from more standard models of production under risk conditions, where risk-decreasing inputs may be used to hedge against production risk, by using more of these inputs than in the risk-neutrality case. Of course, in our case, the choice of $X$ will nevertheless affect the wealth level, while parameter $\alpha$ would affect both productivity and the level of production risk independently from agent's actions.

${ }^{6}$ Note that we restrict our attention to the case of non stochastic, continuous and piecewise differentiable contracts. Stochastic contracts may perform better in this framework, as they could help reducing the incentive cost, albeit with the drawback of insurance costs.

${ }^{7}$ We follow Laffont and Rochet by assuming that ex-post participation constraints imply the ex-ante participation constraint that we neglect hereafter.

${ }^{8}$ Note that because $V_{f}=p$, we have $S_{Z}=0$ and $S_{X}=-D_{X}$.

${ }^{9}$ Unit corn output price and nitrogen input price are set to their empirical means: $p=71.4$ and $w=3.50$. Simulation results were not significantly different for alternative values of $A$ and $\gamma$.

${ }^{10}$ We check that the crop yield function $F($.$) is globally concave and also concave in X$ and $Y$. Since the expression we will use in the sequel for this function depends on $Z$ instead of $Y$, we also check for the concavity of $f(\alpha, \gamma, A, X, Z)$. Finally, the cross product between $\alpha$ and $Z$ appears to be negative in the latter, so that the assumption $\partial^{2} f / \partial \alpha \partial Z<0$ is not invalidated.

${ }^{11}$ The value for $\rho$ is close to the one we have estimated for the same crop on a different dataset (see Bontems and Thomas).

${ }^{12}$ Maximum $\rho$ was set to 5 , as results were stable for higher values of this parameter.

${ }^{13}$ We do not attempt to model this issue here, which is outside the scope of this article.

${ }^{14}$ We will have to verify that local second order conditions are satisfied, i.e. $\int_{\underline{\alpha}}^{\bar{\alpha}}\left[\frac{\partial^{2} U(\Pi(\alpha))}{\partial X^{2}}\right] d G(\alpha) \leq 0$ Actually, this condition is checked under our assumption $\frac{\partial^{2} f}{\partial X^{2}} \leq 0$. 


\section{References}

Babcock, B.A. and D.A. Hennessy. "Input Demand under Yield and Revenue Insurance." American Journal of Agricultural Economics 78(1996):416-27.

Babcock, B.A. "The Effects of Uncertainty on Optimal Nitrogen Applications." Review of Agricultural Economics 14(1992):271-80.

Baron D. and R. Myerson. "Regulating a Monopolist with Unknown Costs." Econometrica 50(1982):911-30.

Bontems, Ph. and A. Thomas. "Information Value and Risk Premium in Agricultural Production: The case of Split Nitrogen Application for Corn." American Journal of Agricultural Economics 82(2000):59-70.

Boswell, F.C., J.J. Meisinger, and N.L. Case. "Production, Marketing, and Use of Nitrogen Fertilizers." Fertilizer Technology and Use. O.P. Engelstad ed. pp. 229-92. Madison WI: Soil Science Society of America, 1985.

Davidson, R. and J.G. MacKinnon. Estimation and inference in econometrics. Oxford: Oxford University Press, 1993.

Dilz, K. "Efficiency of Uptake and Utilization of Fertilizer Nitrogen in Plants." Nitrogen Efficiency in Agricultural Soils. D.S. Jenkinson and K.A. Smith eds. pp. 1-26. London: Elsevier, 1988.

Feinerman, E., E.K. Choi, and S.R. Johnson. "Uncertainty and Split Nitrogen Application in Corn Production." American Journal of Agricultural Economics 72(1990):975-84.

Guesnerie R. and Laffont J.J. "A complete solution to a class of principal agent problems with an application to the control of a self-managed firm". Journal of Public Economics 25(1984):329-69.

Hennessy, D.A. and J. Roosen. "Tests for the Role of Risk Aversion on Input Use." American Journal of Agricultural Economics 85(2003):30-43.

Huang, W., N.D. Uri, and L. Hansen. "Timing Nitrogen Fertilizer Applications to Improve Water Quality.” USDA Staff Report No. 9407, Washington DC, 1994. 
Laffont J.J. and J.C. Rochet. "Regulation of a risk averse firm." Games and Economic Behavior 25(1998):149-73.

Laffont J.J., Tirole J. A theory of incentives in procurement and regulation. Cambridge: The MIT Press, 1993.

Lambert, D.K. "Risk Considerations in the Reduction of Nitrogen Fertilizer Use in Agricultural Production." Western Journal of Agricultural Economics 15(1990): $234-44$.

Leathers, H.D., and J.C. Quiggin. "Interactions between Agricultural and Resource Policy: The Importance of Attitudes Toward Risk." American Journal of Agricultural Economics 73(1991):757-64.

Meisinger, J.J., and G.W. Randall. "Estimating Nitrogen Budgets for Soil-Crop Systems." Managing Nitrogen for Groundwater Quality and Farm Profitability. R.F. Folley, D.R. Keeney and R.M. Cruse eds. pp. 85-124. Madison WI: Soil Science Society of America, 1991.

Moschini, G. and D.A. Hennessy. "Uncertainty, risk aversion and risk management for agricultural producers." Handbook of agricultural economics. B.L. Gardner and G.C. Rausser eds. pp. 87-153. Amsterdam: Elsevier Science, 2001.

Pierce, F.J., M.J. Shaffer and A.D. Halvorson. "Screening procedure for Estimating Potentially Leachable Nitrate-Nitrogen Below the Root Zone." Managing Nitrogen for Groundwater Quality and Farm Profitability. R.F. Folley, D.R. Keeney and R.M. Cruse eds. pp. 259-84. Madison: Soil Science Society of America, 1991.

Polman, N.B.P. and G.J. Thijssen. "Combining results of different models: the case of a levy on the Dutch nitrogen surplus." Agricultural Economics 27(2002):41-49.

Ramaswami, B. "Production Risk and Optimal Input Decisions." American Journal of Agricultural Economics 74(1992):860-869.

Salanié, B. "Sélection adverse et aversion pour le risque". Annales d'Economie et de Statistique 18(1990):131-49. 
SCEES. "The 1994 Cropping Practices Survey." Paris: Ministry of Agriculture, 1998.

Segerson, K. "Uncertainty and Incentives for Nonpoint Pollution Control." Journal of Environmental Economics and Management 15(1988):87-98.

Wu J.J. and B.A. Babcock. "Contract design for the purchase of environmental goods from agriculture." American Journal of Agricultural Economics 78(1996):935-45. 
Table 1. Descriptive Statistics on the Sample

\begin{tabular}{lrrrrr}
\hline Variable & Unit & Mean & Standard deviation & Minimum & Maximum \\
\hline Crop yield & q/ha & 88.6077 & 24.2695 & 22.00 & 151.00 \\
$X$ & $\mathrm{~kg} / \mathrm{ha}$ & 65.7142 & 38.5540 & 5.00 & 309.00 \\
$Y$ & $\mathrm{~kg} / \mathrm{ha}$ & 130.1065 & 55.8326 & 14.00 & 416.00 \\
\hline
\end{tabular}

Note: $\mathrm{N}=341$. One quintal per ha $(\mathrm{q} / \mathrm{ha})$ is about 220.46 pounds per hectare. 
Table 2. Instrumental Variable Parameter Estimates

\begin{tabular}{lrrc}
\hline Variable & Estimate & Std. Error & p-value \\
\hline Constant & 63.1072 & 16.1693 & 0.0001 \\
$X$ & 0.3905 & 0.1728 & 0.0240 \\
$Y$ & 0.4284 & 0.1825 & 0.0189 \\
$X^{2}$ & -0.0011 & 0.0005 & 0.0610 \\
$Y^{2}$ & -0.0001 & 0.0010 & 0.8835 \\
$X \times Y$ & -0.0033 & 0.0013 & 0.0119 \\
\hline
\end{tabular}

Note: $\mathrm{N}=341 . R^{2}=0.1232$. Fisher test statistic is 2.66 (p-value 0.0240 ). 
Table 3. Simulation Results, Status Quo Case Only

\begin{tabular}{lrrrrrr}
\hline \hline & & & & & & \\
$\rho$ & $X$ & $E[D]$ & $E(Z)$ & $E(\Pi)$ & $C E(\Pi)$ & Total surplus \\
\hline 0 & 144.89 & 778.19 & 235.22 & 445.39 & 445.39 & -332.79 \\
0.5 & 141.81 & 751.81 & 233.01 & 445.36 & 440.07 & -311.73 \\
2.0 & 131.72 & 668.46 & 225.76 & 444.87 & 428.69 & -239.76 \\
3.0 & 115.89 & 547.50 & 214.38 & 442.86 & 417.13 & -130.37 \\
4.0 & 78.21 & 308.21 & 187.30 & 432.02 & 397.80 & 89.58 \\
5.0 & 62.51 & 228.66 & 176.02 & 424.98 & 390.83 & 162.17 \\
\hline \hline
\end{tabular}

Note: $X$ is first-period nitrogen application; $D$ is the environmental damage function; $E(Z)$ and $E(\Pi)$ are expected nitrogen use and profit (per hectare) respectively; $C E(\Pi)$ is Certainty Equivalent of $\Pi$. Values are in Euros per hectare, except for $X$ and $E(Z)$ (in $\mathrm{kg} \mathrm{N} / \mathrm{ha}$ ). 
Table 4. Simulation Results and Social Surplus Calculations

\begin{tabular}{crrrrrr}
\hline \hline & $X$ & $E[D]$ & $E(Z)$ & $E[(1+\lambda) T]$ & $C E(\Pi)$ & Total surplus \\
\hline Perfect Information & 18.94 & 69.98 & 207.56 & -493.54 & 0 & 423.55 \\
Risk neutrality & 39.06 & 131.92 & 39.06 & 93.71 & 184.70 & -40.93 \\
CRRA $\rho=0.5$ & 41.05 & 139.08 & 42.33 & 166.63 & 271.67 & -34.03 \\
$\rho=2.0$ & 61.08 & 222.00 & 68.21 & 69.74 & 292.63 & 0.89 \\
$\rho=3.0$ & 69.88 & 264.53 & 95.09 & -5.98 & 277.46 & 18.90 \\
$\rho=4.0$ & 74.28 & 287.19 & 135.54 & -82.49 & 232.26 & 27.56 \\
$\rho=5.0$ & 74.29 & 287.23 & 146.68 & -100.18 & 213.04 & 25.99 \\
\hline \hline
\end{tabular}

Note: $X$ is first-period nitrogen application per hectare; $D$ is the environmental damage function; $Z$ is total nitrogen per hectare; $(1+\lambda) T$ is transfer weighted by the opportunity cost of public funds; $C E(\Pi)$ is Certainty Equivalent of $\Pi$.. CRRA is constant relative risk aversion; Values are in Euros per hectare, except for $X$ and $E(Z)$ (in $\mathrm{kg} \mathrm{N} / \mathrm{ha}$ ). 
Contract $(\mathrm{T}, \mathrm{Z})$ is signed

Action $X$ is taken
Action $Y$ is taken

and profit is obtained

\begin{tabular}{|c|c|c|c|}
\hline$\downarrow$ & $\downarrow$ & $\alpha$ is observed & $\downarrow$ \\
\hline$t=0$ & $t=1$ & $\begin{array}{c}t=2 \\
\uparrow \\
\hat{\alpha} \text { is announced }\end{array}$ & $t=3$ \\
\hline
\end{tabular}

Figure 1. The timing of the game 


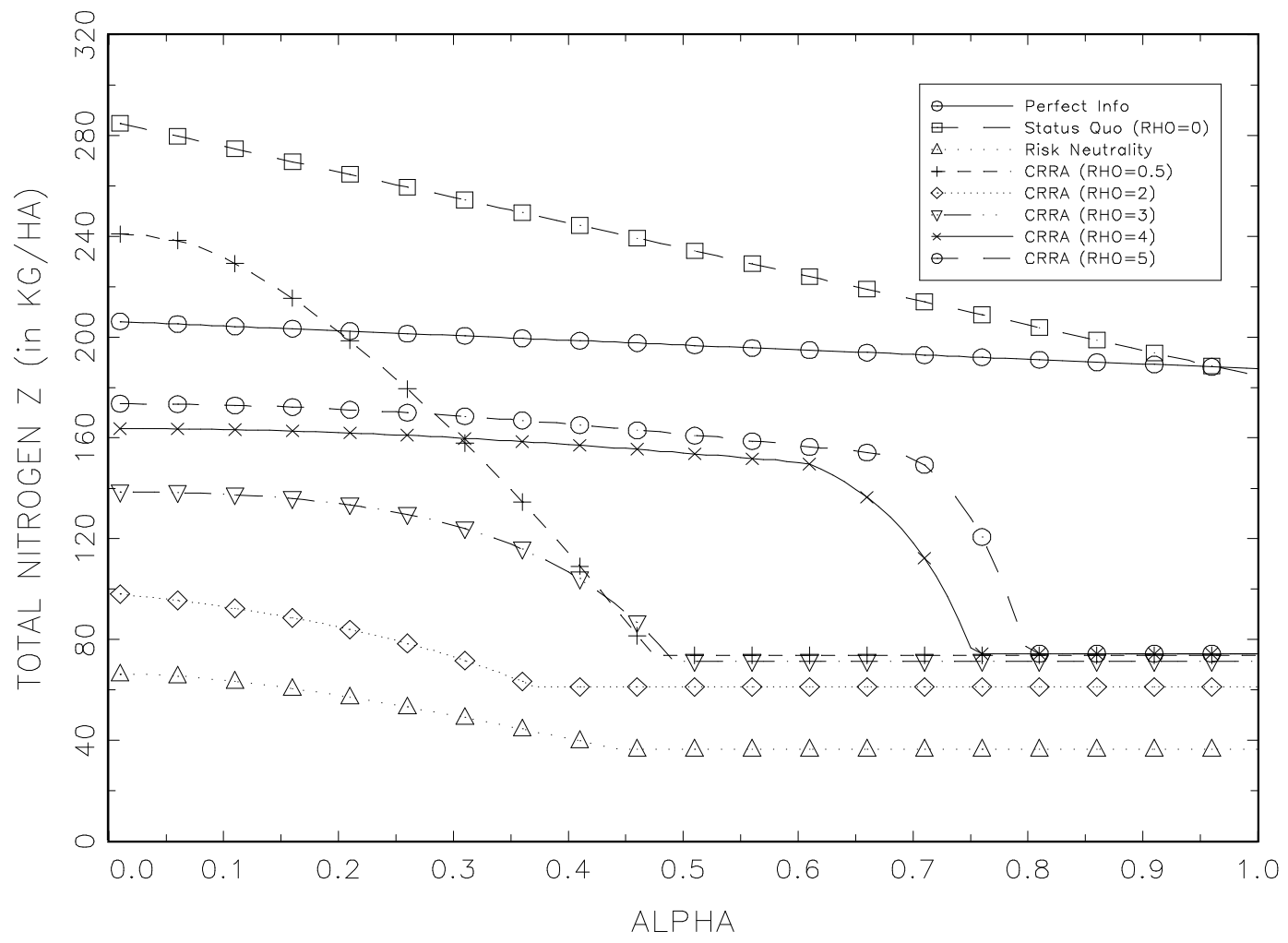

Figure 2. Optimal path for nitrogen quota $Z(\alpha)$ 


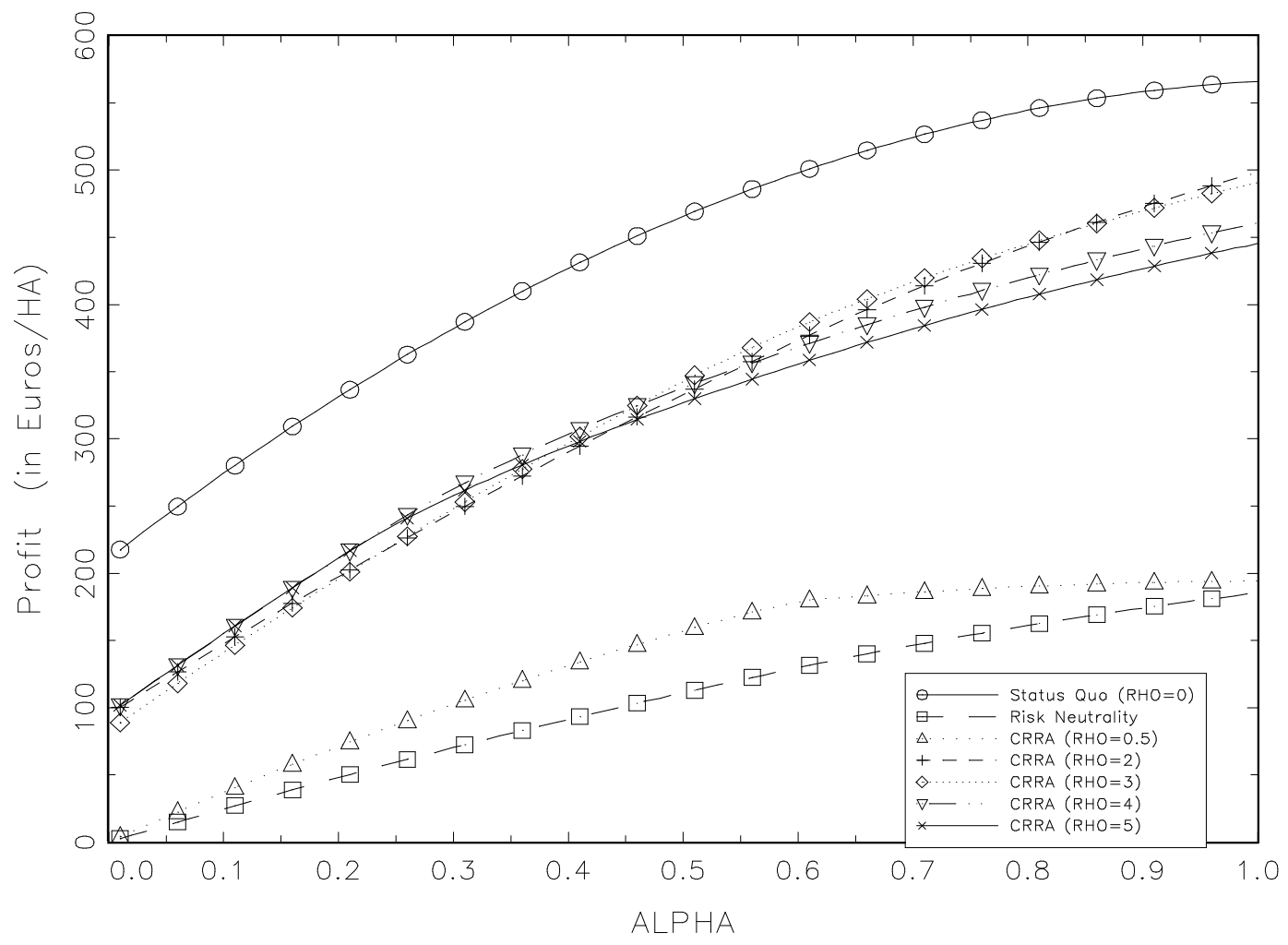

Figure 3. Profit $\Pi(\alpha)$ 